\title{
Imaging silicon by atomic force microscopy with crystallographically oriented tips
}

\author{
F.J. Giessibl*, S. Hembacher, H. Bielefeldt, J. Mannhart \\ Universität Augsburg, Institute of Physics, Electronic Correlations and Magnetism, Experimentalphysik VI, Universitätsstrasse 1, 86135 Augsburg, \\ Germany
}

Received: 16 July 2000/Accepted: 14 December 2000/Published online: 27 March 2001 - @ Springer-Verlag 2001

\begin{abstract}
The images obtained by atomic force microscopy (AFM) originate from a convolution of atomic tip and sample states. Since the vertical resolution of AFM is approaching the picometer level, the atomic and subatomic structure of the tip is becoming increasingly important. Here, we demonstrate the preparation of crystallographically oriented AFM tips by breaking a silicon wafer along its preferential cleavage planes. Assuming bulk termination, the front atom of this tip should expose a single dangling bond. Images derived with this tip are consistent with this speculated tip geometry and show unprecedented vertical distinction of the six different surface atom sites of the $\operatorname{Si}(111)-(7 \times 7)$ structure.
\end{abstract}

PACS: 07.79.Lh; 34.20.Cf; 68.37.E

Recently, sub-atomic resolution on the surface of silicon $(111)-(7 \times 7)$ with frequency-modulation atomic force microscopy (FM-AFM) [1] has been demonstrated. The subatomic structure of the adatom images was attributed to a tip with a very special symmetry: a tip with a front atom exposing two unsaturated bonds. The experimental observation at subatomic resolution outlines the importance of both the chemical nature and the geometric orientation of the front atom with respect to its nearest neighbors in the tip. While the preparation of the silicon sample to obtain the $7 \times 7$ reconstruction is straightforward, the preparation of the tip is more challenging. Here, we report on the construction of a deliberately shaped tip with predetermined crystallographic orientation.

Most force sensors for AFM are microfabricated, and the crystallographic orientation of the tip is determined by the manufacturing process of the whole cantilever. Typically, the tips of micromachined cantilevers point in the $\langle 100\rangle$ direction [2] and are only a few micrometers high. Since micromachined cantilevers are etched out of single crystals, the angle

${ }^{*}$ Corresponding author. (E-mail: franz.giessibl@physik.uni-augsburg.de)

NC-AFM 2000 - Third International Conference on Non-Contact Atomic Force Microscopy, July 16-19, 2000 in Hamburg, Germany of the crystallographic orientation between tip and sample is given by the tilt angle of the cantilever. To assure that the micromachined tip is closer to the flat sample than any other parts of the cantilever and mounting devices, tilt angles between $10^{\circ}$ and $25^{\circ}$ typically have to be applied. The angle between the orientations of tip and sample cannot be freely chosen. This constraint does not apply to force sensors based on a tuning fork ("qPlus" sensor, see Fig. 1 in [3]), since it is possible to attach large tips on these sensors with a deliberate crystallographic orientation.

The natural cleavage planes of silicon are (111) planes, since the free surface energy is minimal for this orientation. Each $\mathrm{Si}-\mathrm{Si}$ bond has a bonding energy of approximately $2.3 \mathrm{eV}$, and the density of unsaturated bonds (dangling bonds) is $7.8 \mathrm{~nm}^{-2}$ for the (111) orientation and $13.6 \mathrm{~nm}^{-2}$ for the (100) orientation. Therefore, silicon exhibits a very strong preference to form (111) limited planes as boundaries of mesoscopic structures. This is nicely evident in the work of Baumgärtner et al., where silicon has been deposited by molecular beam epitaxy onto a Si (100) wafer through a square shaped $350 \mathrm{~nm} \times 350 \mathrm{~nm}$ aperture. The resulting structure was a pyramid with (111) plane boundaries, rather than a pile with a square cross section (Fig. 4.7 in [4]). Figure 1a shows a model of a silicon cluster built from a chemist's model kit (Part No. 660710, Leybold Didactic GmbH, Hürth, Germany) with atoms with tetrahedral symmetry. It is interesting to note that the corner atoms of a silicon cluster which is bounded by (111) planes must expose two dangling bonds per corner atom, unless one of the (111) planes is only one atom wide. In this case, it is also possible to orient the cluster such that the corner atom exposes a single dangling bond (indicated with a " $t$ " in Fig. 1a).

The material we used for our tips was a (111) oriented Si wafer with a diameter of $76.2 \mathrm{~mm}$, a thickness of $490 \mu \mathrm{m}$ and a resistivity of $9.0 \Omega \mathrm{cm}$ (p-type, Boron doped). Etching silicon in $\mathrm{KOH}$ yields highly anisotropic etching rates, with (111) planes yielding by far the slowest etch rates [5]. Thus, by etching macroscopic pieces of silicon it should be possible to obtain clusters like that shown in Fig. 1a. However, etching pieces to sharpen them has so far not resulted in smooth planes. Possibly the concentration of dopants was too 


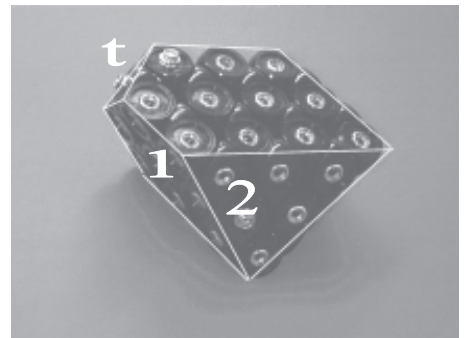

a

Fig. 1. a Model of a silicon cluster bounded by (111) planes. Two (111) planes are identified by "1" and "2" and the tip is identified by " $\mathrm{t}$ ". b Photograph of the silicon tip of a qPlus sensor next to a silicon sample. The planes labelled " 1 " and " 2 " correspond to the planes in a. Plane 2 is an original surface plane of the wafer, and the plane which is hidden by plane 1 is parallel to plane 2 and also an original surface plane. Thus, the front atom is expected to expose a single dangling bond towards the sample if the (111) planes continue all the way to the tip

high in the material used so that the etching planes were not smooth. Therefore, we tried a different approach. We crushed silicon (111) wafers and investigated the broken pieces under an optical microscope. While some pieces showed a large step density, several chunks could be identified which were limited by large and very smooth planes. The angles of the cleavage planes with respect to the front and back sides (plane 2 in Fig. 1b) were $55^{\circ}$; thus, these cleavage planes could be identified as (111) planes. Figure $1 \mathrm{~b}$ is a photograph of such an oriented tip showing both the tip and its mirror image reflected from the silicon sample. The numbers " 1 " and "2" in Fig. 1a and b show the correspondence between the (111) planes in the model and the real tip. The orientation of this tip was such that the front atom should have a single $s p^{3}$ orbital pointing towards the sample, provided that the (111) planes continue all the way to the end of the tip. We mounted this tip on top of a qPlus force sensor and introduced the sensor into an ultra-high vacuum environment. Initially, the tip did not work well in scanning tunneling microscopy (STM) mode on a silicon sample. Possibly, the native oxide prevented stable tunneling conditions. However, after heating the tip by electron bombardment, the tip worked excellently in STM mode. Conventional cantilevers are also cleaned by in situ sputtering [6] or heating [7]. When the tip is heated to remove the oxide, minimal free surface energy might be obtained not by tips where the (111) orientation extends all the way to the front atom, but by a rounded apex consisting of higher indexed facets at the tip apex, such as proposed by Arai and Tomitori [8].

After switching to FM-AFM mode, excellent AFM data was obtained; in agreement with the expected symmetry of the front atom, single maxima for each adatom were observed (Fig. 2). This image was taken in the topographic mode with the following parameters: oscillation amplitude $A=0.3 \mathrm{~nm}$, spring constant $k=1800 \mathrm{~N} / \mathrm{m}$, eigenfrequency $f_{0}=20531 \mathrm{~Hz}$ and frequency shift $\Delta f=+85 \mathrm{~Hz}$. Imaging with positive frequency shifts does not necessarily mean that the tip sample force was repulsive [9]. Both the adatoms and the restatoms are visible in Fig. 2. While the local maxima at the restatom positions (i.e. between corner atoms and two neighboring center atoms) are not very pronounced, the local minima between the three center atoms are clearly visible in both the faulted and unfaulted halves. It is worthy to note that
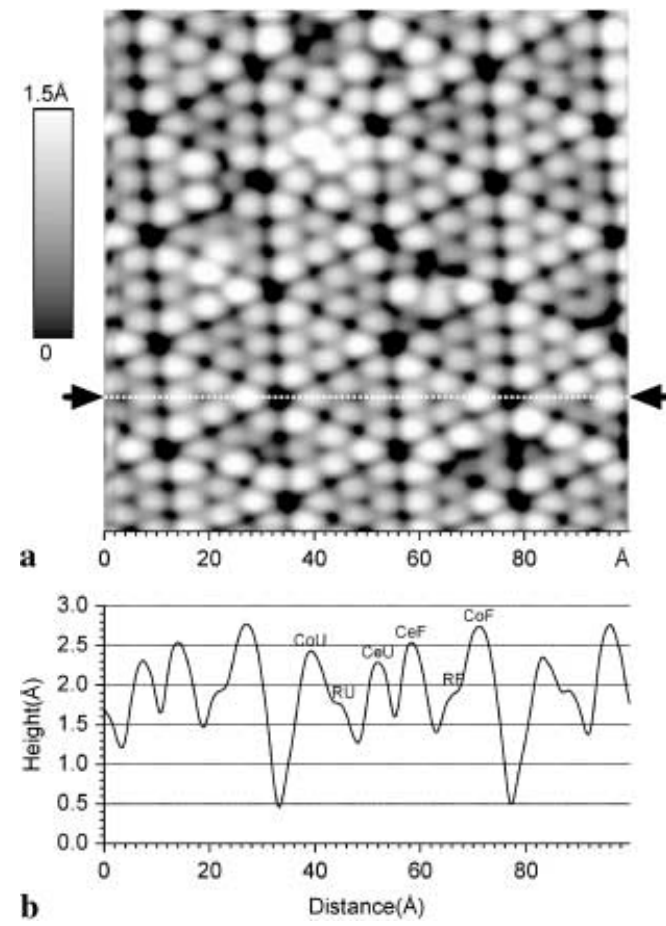

Fig. 2. a Noncontact AFM image of $\mathrm{Si}(111)-(7 \times 7)$ obtained with the following imaging parameters: $A=0.3 \mathrm{~nm}, k=1800 \mathrm{~N} / \mathrm{m}, f_{0}=20531 \mathrm{~Hz}$ and $\Delta f=+85 \mathrm{~Hz}$. b Profile of a along the long diagonal of the $\mathrm{Si}(111)-$ $(7 \times 7)$ unit cell

the cornerhole depth is $0.23 \mathrm{~nm}$, considerably deeper than all other AFM results known to us at this time. Tomitori and Arai suggested a rounding effect when heating Si tips due to the formation of (103), (113) and (110) facets [8]. If faceting had occurred in our experiment, the tip would not have been able to penetrate as far into the cornerholes as measured. We therefore presume that the temperature reached while heating the tip was high enough to remove the oxide and low enough to prevent faceting. In lack of a more sophisticated tip model which is compatible with our observations, we propose that our tip might be terminated by the bulk configuration, i.e. with a single front atom.

The positions of the surface atoms in the Si $(111)-(7 \times 7)$ reconstruction have been studied experimentally by lowenergy electron diffraction (LEED) [10] as well as theoretically [11]. The 12 adatoms and 6 restatoms within one unit cell belong to 4 and 2 different symmetry types respectively. Table 1 shows the relative heights of the surface layer atoms derived by calculations using LEED and AFM measurements.

While the relative heights derived by different techniques show large discrepancies, at least the sequence of the heights is consistent for the LEED data - and the AFM data from our group. The restatoms in the $\mathrm{Si}(111)-(7 \times 7)$ reconstruction were first observed by Lantz et al. approximately $40 \mathrm{pm}$ below the adatoms [13]. The z-position of the restatoms according to our new AFM data is almost exactly the same as that for the LEED data.

In summary, we have shown that it is possible to obtain atomic resolution using AFM with cleaved macroscopic silicon tips. These tips can be oriented in any crystallographic orientation, which allows detailed study of the bonding symmetry between silicon tips and samples. While at this time we 
Table 1. Relative heights (pm) of the surface atoms of $\mathrm{Si}(111)-(7 \times 7)$ with respect to the corner adatom in the faulted half as obtained from calculations using LEED and AFM measurements. CoF: corner adatom, faulted; CeF: center adatom, faulted; RF: rest atom, faulted; CoU: corner adatom, unfaulted; CeU: center adatom, unfaulted; RU: rest atom, unfaulted

\begin{tabular}{crrcccc}
\hline Type & Calc. [11] & LEED [10] & AFM [12] & AFM [13] & AFM [1] & Present AFM data \\
\hline $\mathrm{CoF}$ & 0 & 0 & 0 & 0 & 0 & 0 \\
$\mathrm{CeF}$ & -5.3 & -4 & 13 & - & -15 & -20 \\
$\mathrm{RF}$ & -103.3 & -101 & - & $\sim-40$ & - & -80 \\
$\mathrm{CoU}$ & -4.6 & -8 & 0 & - & -19 & -31 \\
$\mathrm{CeU}$ & -8.4 & -12 & 13 & - & -34 & -46 \\
$\mathrm{RU}$ & -100.7 & -106 & - & $\sim-40$ & - & -97 \\
\hline
\end{tabular}

cannot be sure that the crystal symmetry of the bulk extends all the way to the end of the tip, the data are compatible with this speculation.

Acknowledgements. We wish to thank T. Arai, C. Quate, L. Scandella, M. Tomitori and M. Tortonese for valuable discussions. This work is supported by BMBF Grant 13N6918/1.

\section{References}

1. F.J. Giessibl, S. Hembacher, H. Bielefeldt, J. Mannhart: Science 289, $422(2000)$

2. O. Wolter, T. Bayer, J. Greschner: J. Vac. Sci. Technol. B 9, 1353 (1991)
3. F.J. Giessibl: Appl. Phys. Lett. 76, 1470 (2000)

4. H. Baumgärtner, W. Hansch, F. Wittmann, I. Eisele: Curr. Top. Cryst. Growth Res. 2, 283 (1995)

5. K.E. Petersen: Proc. IEEE 70, 420 (1982)

6. P. Güthner: J. Vac. Sci. Technol. B 14, 2428 (1996)

7. T. Arai, M. Tomitori: Appl. Surf. Sci. 157, 207 (2000)

8. T. Arai, M. Tomitori: J. Vac. Sci. Technol. B 18, 648 (2000)

9. F.J. Giessibl: submitted to Appl. Phys. Lett. (2001)

10. S.Y. Tong, H. Huang, C.M. Wei, W.E. Packard, F.K. Men, G. Glander, M.B. Webb: J. Vac. Sci. Technol. A 6, 615 (1988)

11. K.D. Brommer, B.E. Larson, M. Needels, J.D. Joannopoulos: Jpn J. Appl. Phys. 32, 1360 (1993); Phys. Rev. Lett. 68, 1355 (1996)

12. R. Erlandsson, L. Olsson, P. Martenson: Phys. Rev. B 54, R8309 (1996)

13. M.A. Lantz, H.J. Hug, P.J.A. von Schendel, R. Hoffmann, S. Martin, A. Baratoff, A. Abdurixit, H.-J. Güntherodt, C. Gerber: Phys. Rev. Lett. 84, 2642 (2000) 